\title{
Compensating the Computational Bias of Spreadsheets with MKM Techniques
}

\author{
Andrea Kohlhase and Michael Kohlhase \\ German Center for Artificial Intelligence (DFKI), Bremen, Germany \\ \{Andrea,Michael\}.Kohlhase@dfki.de
}

\begin{abstract}
Spreadsheets are mathematical documents that are heavily employed in administration, financial forecasting, education, and science because of their intuitive, flexible, and direct approach to computation. In this paper we show that spreadsheets are interesting applications for MKM techniques which can alleviate usability and maintenance problems as spreadsheet-based applications grow evermore complex and longlived. We present the software and information architecture of a semantic enhancement of MS Excel spreadsheets that aims at compensating the computational bias in spreadsheets.
\end{abstract}

\section{Introduction}

Spreadsheets programs are mathematical software systems: they contain mathematical formulae and are used in financial forecasting, education, and science because of their intuitive, flexible, and direct approach to mathematical computation. It has been estimated that each year tens of millions professionals and managers create hundreds of millions of spreadsheets [Pan00]. This probably makes spreadsheets the most heavily used mathematical software systems at this point of time and should therefore be an interesting testbed for MKM applications. But it seems that the MKM community has not risen to this opportunity; possibly since the mathematical aspects are geared almost exclusively to computational concerns and the declarative aspects of mathematical knowledge that are the concern of the MKM community seem to play a subordinate role in spreadsheets at first glance.

In this paper we show that semantic knowledge management techniques can be used to enhance the interaction with spreadsheets and alleviate usability problems appearing with spreadsheet complexity: in many spreadsheet-based applications even longtime users cannot interpret all data and are not certain about their origins (see [AE06] and its references for a discussion), which often results in errors on the data level and misinterpretation or misapprehension of the underlying model.

In the next section we will start out with an analysis of the semantic layers of spreadsheets. To compensate the diagnosed computational bias we propose to augment the two existing semantic layers of a spreadsheet - the surface structure and the formulae - by a third that makes the intention of the spreadsheet 
author explicit. In the SACHS project we encode the intention as an accompanying OMDoc [Koh06] document and can thereby provide multi-layered, semantic help services.

\section{Semantic Layers in Spreadsheets}

Instead of developing the general theory, we will expose the salient parts of our approach using WiNOGRAD's example spreadsheet from [Win06] (re-represented in MS Excel in Figure 1 on page 3) as a running example. We will differentiate the three semantic layers in turn and draw conclusions viewing this spreadsheet as both an active and a semantic document.

\subsection{Active and Semantic Documents}

We call a document semantic, iff it contains an infrastructure that distinguishes between content and form. Note that such an infrastructure can range from superficial styling information in PowerPoint slide masters or $\mathrm{L}_{\mathrm{E}} \mathrm{X}$ document classes, over RDFa [W3C08] annotations in web pages to formal logic specifications of program behaviors. The idea is that this infrastructure makes relations between the objects described in the document explicit, so that they can be acted upon by machines. In particular, semantic documents can be interpreted by "presentation engines" that operationalize the semantic relations by allowing the reader to interact with various aspects of the semantic properties. We call the combination of a semantic document with a presentation engine that can adapt the surface structure of the document to the environment and user input an active document. Our definition is between the concept of embedding semantic networks into hyper-documents employed by GaINES and SHAw in [GS99] and the rather visionary notion of documents that can answer arbitrary questions about their content proposed by HEINRICH and MAURER [HM00]. Crucially both presuppose some kind of content representation in or near the document and a suitable "presentation engine".

For the purposes of this paper, we will neglect the fact that most presentation engines also incorporate editing facilities and concentrate on the interaction with active documents for reading and exploration. This view is similar to what $\left[\mathrm{UCI}^{+} 06\right]$ call "intelligent documents".

A paradigmatic example of an active document is a Mathematica notebook [Wol02], where equations and mathematical objects can be inspected, visualized, and manipulated by the user. Here, the semantic document is written in the MATHEMATiCA markup language which includes a content markup scheme for mathematical formulae and a high-level programming language. The presentation engine is the Mathematica front-end which presents interactive documents to the user and calls the Mathematica kernel for evaluation of program fragments and computation of mathematical properties.

Spreadsheets are another paradigmatic class of active documents. Here the semantic document contains representations of the cell values or formulae together with display information such as cell color, font information, and current 
viewport. The presentation engine is a spreadsheet program like MS Excel, which presents the semantic document to the user by giving it a grid layout and recalculates values from formulae after each update. But what is the underlying semantic model, i.e. what is the "activeness" of spreadsheets based on?

\subsection{The Surface/Data Layer}

If we look at the example in Figure 1, we see that the grid of cells can be roughly divided into three areas. The darker, ochre area in the center contains values of actual and past expenses and revenues; the lighter, yellow box on the right contains values projected from these. The white region that surrounds both boxes supplies explanatory text or header information that helps users to interpret these numbers. Generally, non-empty cells that do not contain input or computed values usually contain text strings that give auxiliary information on the cells that do; we call these cells collectively the legend of the spreadsheet, since they serve the same purpose as the legend of a map.

Observe that row 17 displays the central values of the spreadsheet: the profit/loss situation over time (i.e., in the years 1984-1988 as indicated by the values in row 4). Moreover note that the meaning of the values in row 17 is that they represent profits and losses as a function $\pi$ of time: recall that a function is a right-unique relation - i.e., a set of pairs of input values and output values.

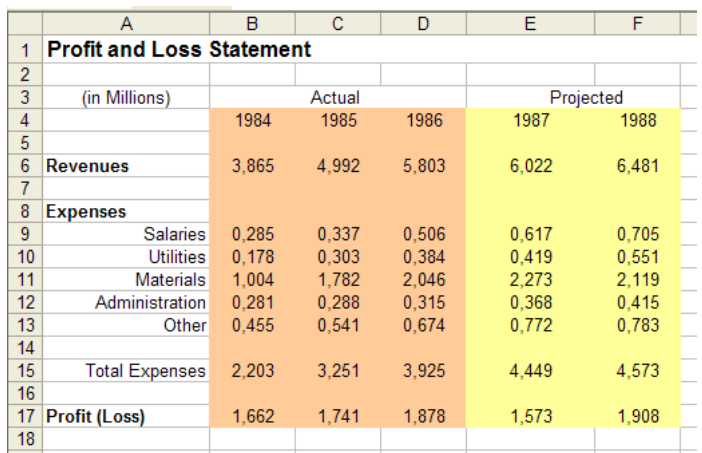

Fig. 1. A Simple Spreadsheet after [Win06] In our example the pair $\langle 1984,1.662\rangle$ of values of the cells [B4] and [B17] is one of the pairs of $\pi$. We will call such a grid region a functional block, and the function it corresponds to its intended function. Empirically, all non-legend, semantically relevant cells of a spreadsheet can be assigned to a functional block, so we will speak of the functional block and the intended function of a cell.

Often a functional block consists of multiple rows and columns and represents a binary function whose values depend on two (main) parameters which are usually in the cells of the column on the left and the row on top of the block. In our example the block with cells [B9:D13] represents a binary function that ranges over expense categories (given in [A9:A13]) and years (given in [B4:D4]). In the general case, the intended function of a functional block can have any arity; its arguments again correspond to functional blocks, which we call input blocks.

Our notion of a functional block is related to, but different from ABRAHAM and ERWIG's of a "table" [AE04,AE06], which also contains row and column header and footer blocks. Our functional blocks roughly correspond to their "table core", whereas we would consider their header blocks as input blocks or 
legend cells (but there may be non-header input blocks in our model) and their footer blocks which contain aggregation cells as separate functional blocks.

\subsection{The Formula Layer}

A spreadsheet cell $c$ may not only be associated with a simple data item (or value) $\llbracket c \rrbracket$, it may also be connected with a formula $\llbracket c \rrbracket$, which evaluates to the cell value. A formula is an expression built up from constants, an extended set of numeric and logic operators, and references to other cells.

In our example, the value of $\pi$ can be computed from the yearly revenues in $\mathcal{R}:=[\mathrm{B} 6: \mathrm{F} 6]$ and the total expenses in $\mathcal{E}:=[\mathrm{B} 15: \mathrm{F} 15]$ by a simple subtraction, the total expenses can in turn be computed by summing up the various particular expense categories listed in cells [A9:A13].

Note that the formulae of cells in a functional block have to be "cp-similar" [BSRR02], i.e., they can be transformed/copied into each other by adjusting the respective rows and columns. We call a functional block computed if all of its formulae are cp-similar. In our example, the functional block $\mathcal{P}:=[\mathrm{B} 17: \mathrm{F} 17]$ is computed: let $\gamma$ range over the columns $B$ to $F$ in $\mathcal{P}$. Note that the formulae $\llbracket \gamma 17 \rrbracket=\gamma 6-\gamma 15$ in cells $[\gamma 17]$ are indeed cp-similar. Together, they make up the function

$$
\mathcal{F}(\mathcal{P}):=\{\langle\llbracket \gamma 6 \rrbracket, \llbracket \gamma 15 \rrbracket, \llbracket \gamma 17 \rrbracket\rangle \mid \gamma \in\{\mathrm{B}, \ldots, \mathrm{F}\}\}
$$

We call $\mathcal{F}(\mathcal{P})$ the function induced by the (formulae in) block $\mathcal{P}$. But we also observe that not all functional blocks in a spreadsheet are computed, for instance the formulae in the block [B9:D13] are all different constants representing the measured values, so they cannot be cp-similar. We call such blocks data blocks and note that the property of being a functional block only depends on a functional correspondence (a conceptual aspect of the data) and not on the existence of formulae (a property of the spreadsheet).

With spreadsheet formulae, users can express data dependencies on a generic level, so that the spreadsheet program can do much computational work in the background. By this virtualization of the traditional ledger sheet (see above), the user's role is lifted to a layman programmer and offers according potential. But ABRAHAM and ERWig report an error rate of up to $90 \%$ (!) in spreadsheets [AE06], which shows that this potential comes with a substantial risk. They analyze the source of many of these errors to be in a mismatch between what the spreadsheet author wants to express and the formulae he writes. They try to address this situation by static analysis techniques (type checking) of the formulae and supplying the author with "spreadsheet templates". To understand this mismatch better, let us now turn to the model the author intends to convey.

\subsection{The Intention Layer}

Note that even though $\mathcal{F}(\mathcal{P})$ and $\pi$ compute the same values, they are completely different functions. $\pi$ is defined via the actual or projected profits or losses of an organization, while $\mathcal{F}(\mathcal{P})$ is a finite, partial binary arithmetic function. Even 
when we compose $\mathcal{F}(\mathcal{P})$ with $\mathcal{F}(\mathcal{R})$ and $\mathcal{F}(\mathcal{E})$ and restrict them to the years 1984-86 yielding $\mathcal{F}:=\left.\mathcal{F}(\mathcal{P}) \circ\langle\mathcal{F}(\mathcal{R}), \mathcal{F}(\mathcal{E})\rangle\right|_{\llbracket \mathrm{B} 4: \mathrm{D} 4 \rrbracket}$, the functions $\mathcal{F}$ and $\pi$ are only extensionally equal (they are equal as input/output relations) and still differ intensionally.

Surprisingly, only $\mathcal{F}$ is explicitly represented in the spreadsheet of Figure 1, moreover, this explicit representation is invisible to the user if she doesn't look at the formula boxes - thus, leaving the user to figure out the 'intention' (the function $\pi$ ) from the implicit information given in the white part by herself. This is why we speak of a computational bias of spreadsheet programs, as some layers of the semantics are explicated but others are not.

Generally, we can assume that spreadsheet program authors use spreadsheets to express and compute (measurable) properties of situations; if we look a little closer then we see that these are not properties of the world as such, but of a high-level, abstract, or mental model of the world, which we subsume under the term intention of the spreadsheet. In our example, the function $\pi$ could be seen as a concept from the intention, whereas the function $\mathcal{F}$ can be seen as its implementation. In our simple example the intention is easy to deduce from the text in the legend and basic financial accounting knowledge.

But even here, some parts of the spreadsheet's intention remain unclear: e.g. for what company or department are the profits and losses computed or what are the methods of projection for the years 1987/8. Let us now take stock of what the cells in the spreadsheet mean and what information we need to infer from this. As we already remarked above, the values of cells [B17:D17] are (the scalar parts of) the actual profits/losses in the years 1984-1986. We need information from cell [A3] for the unit of measurement, from cells [B3:D3] that they are actual, and from [A17] for the interpretation as a 'profit/loss'. To understand the full meaning of these cells, we also need to know about profits and losses of companies - e.g. that high profits of a company I am employed by or that I own stock in are good for me, the fact that the company is based in the Europe and therefore calculates finances in $€$, and that values that are actual are computed from measured values. Finally, we need to know that the profit/loss of an organization over a time interval is defined as the difference between its revenues and expenses over this interval. This knowledge allows to compute the values of cells in $\mathcal{P}$ with the respective formulae from the values of cells in $\mathcal{R} \cup \mathcal{E}$ (i.e., using the function $\mathcal{F}$ ). The values of the cells in $\mathcal{E}$ can be similarly computed from the values of the cells [B9:D13]. Note that while the definition of profits and losses above is general accounting knowledge, this definition is particular to the respective company, as the applicable expenses vary with the organization.

A similar account can be given for the projected profits/losses in cells [E17:F17], only that the interpretation of the cells wrt. the intention is even more diffi-

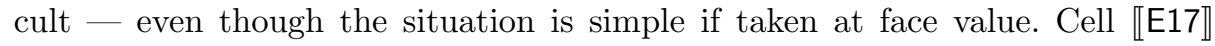
is the projected profit in the year 1987, which is computed from the revenue and expenses in column E. But in contrast to the values in the actual block $[\mathrm{B} 6: \mathrm{D} 6] \cup[\mathrm{B} 9: \mathrm{D} 13]$, the values in the projected block $[\mathrm{E6}: \mathrm{F} 6] \cup[\mathrm{E9}: \mathrm{F} 13]$ are not 
measured, but projected from the actual values by some financial forecasting method that is reflected in the respective formulae. Note that the correspondence of the formula need not be as direct as in the case of the total expenses above. It might be that the forecasting method is defined abstractly, and the concrete formula is derived from it making some simplifying assumptions. Furthermore, to fully understand the values we need to know what assumptions the forecasting method makes, what parameter values are employed and why, how reliable it is, etc. All of these concerns are not addressed at all in the spreadsheet as an active document. Abraham and Erwig describe this situation as follows:

There is a high level of ambiguity associated with spreadsheet template inference since spreadsheets are the result of a mapping of higher-level abstract models in the user's mind to a simple two-dimensional grid structure. Moreover, spreadsheets do not impose any restrictions on how the users map their mental models to the two-dimensional grid (flexibility is one of the main reasons for the popularity of spreadsheets). Therefore the relationship between the model and the spreadsheet is essentially many-to-many [...].

[AE06, p. 5]

\section{Compensating the Computational Bias}

Our analysis of the example above has shown us that large parts of the intention of a spreadsheet is left implicit, even though it is crucial for a user's comprehension. In particular, a user needs to know the following for a spreadsheet:

- The ontology, i.e., background information about relations between concepts and objects of the intention. The objects in the intention include the functions represented in the spreadsheets e.g. $\pi$, their properties, e.g. the units of their arguments and values, and thus of the values in the cells.

- The provenance of data in a cell, i.e., how the value of this data point was obtained, e.g. by direct measurement, by computation from other values via a spreadsheet formula, or by import from another source; see $\left[\mathrm{MGM}^{+} 08\right]$ for a general discussion of provenance.

- The interpretation, i.e., a correspondence between functional blocks and concepts or objects of the intention. We distinguish three parts here

- The functional interpretation, that specifies the intended function of the functional block.

- The value interpretation, i.e. a bijective function that specifies how to interpret the values of the block cells as ontology objects.

- The formula interpretation that links the formulae of a block to an object in the ontology. This mapping must be a refinement in the sense that the interpretations of proper formulae compute the same value as the formulae itself and the pseudo-formulae input is mapped to a provenance object. 
In some spreadsheets that are the digital equivalent to "back-of-the-envelope calculations", the interpretation, provenance, and ontology information is simple to infer, so that the un-documented situation is quite tolerable. Indeed this shows the cognitive strength of the table metaphor, in our example it is no problem for the human reader to interpret the legend item "(in Millions)" as a specification of the value interpretation of the cells [B6:F17] (but not of the years $\mathcal{Y}:=[\mathrm{B} 4: \mathrm{D} 4]$ ).

In many cases spreadsheets have developed into mission-critical tools that are shared amongst whole departments, because they encapsulate important, non-trivial, institutional knowledge. The intention of such spreadsheets is much harder to infer, a fact that is witnessed by the fact that companies spend considerable energy to train employees in the usage (and intention) of such spreadsheets.

In this situation, it would be natural to make spreadsheets even more active to support the user's comprehension of the spreadsheet intention. Thus, in light of the discussion of Section 2.1, we suggest to compensate for the computational bias diagnosed above by extending the underlying semantic document of a spreadsheet. Concretely, we propose to represent the intention (as the provenance and ontology) and to tie the cells in the spreadsheets to concepts in the intention (via an interpretation function).

\subsection{Fixing the Ontology}

We have (at least) two possibilities to extend spreadsheets with an ontology and provenance component: we can either extend spreadsheets by ontology and provenance facilities or we can extend them by interpretation facilities that reference external representations of the intention. As we have seen above, the intention contains quite a lot of information, and making it explicit in a software framework means a large investment. Therefore we contend that an external representation of the intention is more sensible, since it can leverage pre-existing tools and profit from interoperability.

We use the OMDoc format (ㅁppen Mathematical Documents, see [Koh06]) to represent the intention model. OMDoc is an XML-based format for representing semi-formal, semantically structured document collections. It allows to factorize knowledge into "content dictionaries" that serve as constitutive contexts for knowledge elements. OMDoc provides a mathematically inspired infrastructure for knowledge representation: document fragments are classified by their epistemic role as e.g. axioms, definitions, assertions, and proofs and mathematical formulae are represented in the OpenMath $\left[\mathrm{BCC}^{+} 04\right]$ or MATHML $\left[\mathrm{ABC}^{+} 03\right]$ formats. Furthermore, OMDoc provides a strong, logically sound module system based on structured "theories" (content dictionaries (CD) extended by concept inheritance and views) [RK09]. Finally, the language has been extended to deal with units and measurable quantities [HKS06] as a prerequisite for interacting with the physical world. We make use of all of these features for modeling the intentions of spreadsheets. In contrast to other ontology modeling languages like OWL [MvH04], the OMDoc format does not commit to a formal logical language, and therefore lacks a native concept of inference but also does not force 
the author to fully formalize the spreadsheet intention and to work around the expressivity limitations of the underlying logical system. Instead, OMDoc allows to locally formalize elements - and thus provide partial inference - with whatever formal system is most suitable; in our application, we mainly use an formal system for arithmetics as a counterpart for spreadsheet formulae.

For the intention model in our example we divide the background knowledge into theories that inherit functionalities from each other via the imports relation. At the very basis we would have a CD Revenues that defines the concept of the revenue of an organization over a time interval. This theory defines the concept of a binary revenue function $\rho$, such that given an organization $o$ and a natural number $n$ the value $\rho(o, n)$ is the revenue (as a monetary quantity) of $o$ over the span of the year $n(\mathrm{AD})$ in the Gregorian calendar. Note that we use this very naive notion of revenues for didactic purposes only. For instance $\rho$ was chosen as a binary function to highlight that there is no automatic agreement between functional correspondences in the table and objects of the intention. We would be perfectly free to analyze the concepts more thoroughly, embarking into representing monetary systems, theories of time, etc. For the purposes of this paper, we assume that we can either appeal to the intuition of the user or inherit these representations from a suitable foundational ontology.

In the same way we proceed with a CD Expenses, which imports from a CD Salaries. Finally, we build a CD Profits that imports from both. In the OMDoc document pictured in Figure 2 we have summarized some of the relevant CDs and the concepts they introduce.

\subsection{Fixing the Provenance}

We enrich our ontology with provenance information: As we have required the formula interpretation to be a refinement, we need to represent an abstract notion of spreadsheet computation in the ontology. This can be readily done making use of e.g. the CASL libraries [CoF04]. For modeling the provenance of user inputs in spreadsheets, we can be extremely minimalistic, just establishing a stub content dictionary that lifts the concept of "user input" to the ontology level. But in our example we can already see what a more elaborate provenance model could give us: We could specify that the salary values in [B9:F9] are not only user inputs, but really manually copied over from another spreadsheet - the top spreadsheet "Salaries" in Figure 2. To take advantage of this (see details in the next section) we have to develop CDs for provenance, adapting and extending

first formalizations reported on in $\left[\mathrm{MGM}^{+} 08\right]$. As this goes beyond the scope of this paper, we leave this to future work.

\subsection{Fixing the Interpretation}

To interpret the cells in $\mathcal{P}$ for example, we need to

- fix the functional interpretation: Identify that $\mathcal{P}$ and $\mathcal{R}$ form functional blocks with input row $\mathcal{Y}$. 
In Section 2.2 we have already discussed the functional interpretation of $\mathcal{P}$ : it is just the intended function $\pi$. Similarly, we link the revenues block $\mathcal{R}$ with a unary function $\rho(c, \cdot)$, where $c$ is the representation of our example company. Then we have to express the semantic counterpart of the spreadsheet formulae. In our OMDoc format we can simply represent this function as $\lambda y . \rho(c, y)$ in OpenMath or MathML.

- fix the value interpretation: In our example we observe that the values in $\mathcal{P}$ are actually only the scalar parts of measurable quantities, in this case measured "in millions" (of $€$ presumably) according to the spreadsheet legend. Similarly, the (string) values Salaries, Utilities, ... in [A9:A13] have to be interpreted as objects in the ontology. Thus in our example, we choose $i:=\{y \mapsto y(A D)\}$ as the value interpretation of block $\mathcal{Y}$ and $j:=\left\{x \mapsto 10^{6} x €\right\}$ for block $\mathcal{P}$; obviously both are bijective.

- fix the formula interpretation: our example the formulae $\gamma 6-\gamma 15$ in the functional block $\mathcal{P}$ would be linked to the formula $\pi($ year $)=\rho($ year $)-$ $\epsilon($ year $)$ in the Profit/Year definition. Furthermore, we would link $\mathcal{R}$ to a semantic provenance object "imported from Salaries.xsl".

In Figure 2 we show the functional interpretation mappings as red, dotted arrows and the formula interpretation as purple, dot-dash-arrows. The totality of cell interpretations in a spreadsheet induces an associated set of CDs we call the intention model. Note that this is indeed a representation of the intention of this spreadsheet.

We can think of the value interpretations as parser/generator pairs that mediate between the scalar function represented by the formulae in the spreadsheet and the intended function in the intention - which is usually a function between measurable

quantities. In particular the functions $\mathcal{F}$ and $\pi$ are related via the commutative diagram on the right, where the function $\mathcal{F}$ is induced by the spreadsheet formulae as discussed in Section 2.4 above. We see that the three components of the in-

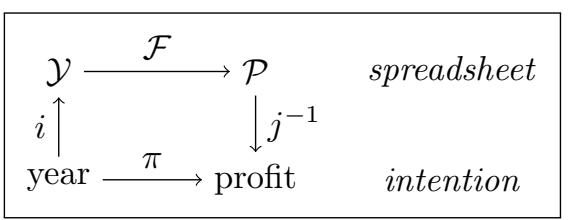
terpretation fully specify the correspondence between functional blocks in the spreadsheet and objects induced by the intention model. To see the strength of this construction let us return to our example and look at the import of salaries from Salaries.xsl. There we have a different value interpretation for the functional block [F6:F6]: this spreadsheet does not calculate in millions, so we chose $k:=\{x \mapsto x\}$ and get the import functions $k \circ j^{-1}=\left\{x € \mapsto 10^{-6} x €\right\}$ in the intention and $j^{-1} \circ k=\left\{x \mapsto 10^{-6} x\right\}$ in the spreadsheet.

In conclusion we observe that setting the ontology, provenance, and the interpretation of a functional block gives us a full and explicit account of its intention, and we can relegate all further semantic services to the intention model. For instance we can verify (using inference in the intention model) this part of the spreadsheet by establishing the equality of $j^{-1} \circ \mathcal{F} \circ j$ and $\rho(c)$. 


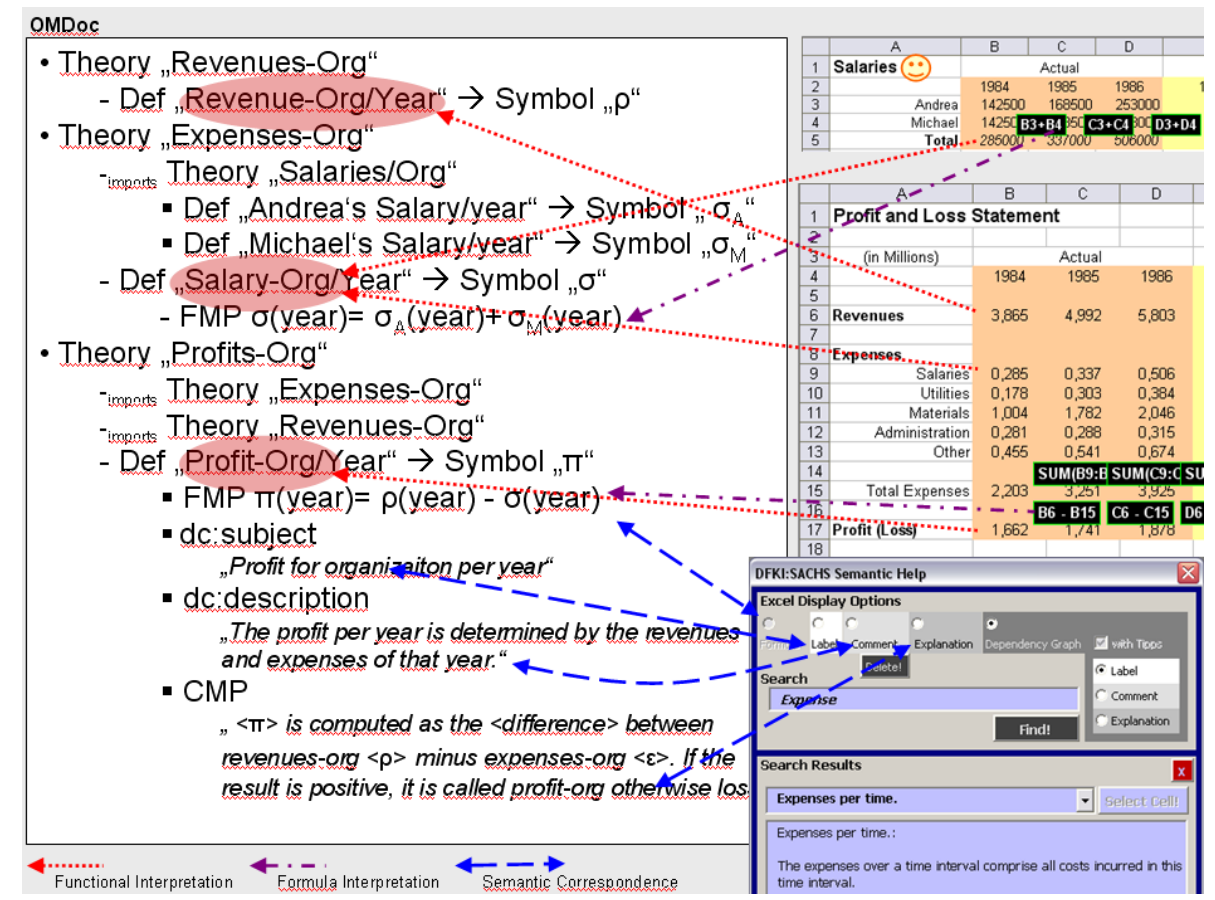

Fig. 2. The SACHS Information Architecture and Control/Search Panel

\section{A Semantic Help System for Spreadsheets with Intentions}

The SACHS system is a work-in-progress add-in for MS Excel (written in Visual Basic for Applications) that aims at providing semantic help facilities for spreadsheets. Though it has been developed for the DFKI controlling system, it works for any spreadsheet whose intention has been encoded as an OMDoc document, e.g. our running example, and mash-up information has been provided ${ }^{1}$. We have designed SACHS as invasive technology that extends well-used (and therefore well-known) software systems from within to overcome usage hurdles see [Koh05b] for a discussion. We designed the semantic services in SACHS to allow the user to explore the intention of a spreadsheet from her micro-perspective, i.e., from her here-and-now. In particular, all semantic help facilities start from the cell level. Moreover, we tried to implement a process-based interaction design, i.e., a design where the semantic help evolves in a user-steered process.

For services that visualize the intention, the cells in the spreadsheet must be interpreted, i.e., linked to elements of the accompanying OMDoc document as e.g. shown in Figure 2. Generally, all cells in a functional block are linked to an OMDoc definition - the definition of its intended function, while OMDoc assertions justify their formulae. This assignment is internally represented by

\footnotetext{
${ }^{1}$ We will spare the reader the technical details of this mash-up for lack of space.
} 
an extra worksheet within the spreadsheet we call the "SACHS interpretation". This is manually maintained by the spreadsheet author. Once the interpretation is established we can directly make use of the various elements of the OMDoc information for the respective objects (see the dashed arrows in Figure 2). Concretely, for instance, the Dublin Core metadata element dc:subject of an OMDoc definition can be used as a SACHS label for the cell it interprets. MS Excel's comment functionality is hijacked to create $S A C H S$ comments that draw on the respective dc:description element, which contains a concise description of the object in question. In contrast, the CMP element of the OMDoc definition contains a detailed explanation using semantically marked up representations of objects from the intention model. These can be mathematical formulae encoded as OpenMath objects like the revenue function $\rho$ or technical terms like "difference" which we have decorated in angle brackets in Figure 2. The added value of semantic annotation here is that the meaning of both can be further explored: The front end item "SACHS explanations" allows this by providing "jump points" from within the text to those cells that are assigned to the definitions of those symbols via the SACHS interpretation sheet. Once jumped the user can look up the available semantic information of that particular cell and so on.

A formula underlying a cell is mirrored in the formula element FMP of the respective definition in the semantic document (see Figure 2) in the OpenMath format, this allows us to present it to the user in math notation: $\sum_{i=1}^{5} \epsilon_{i}(1985)$ is more readable than "=SUM $(\mathrm{C} 9: \mathrm{C} 13)$ ".

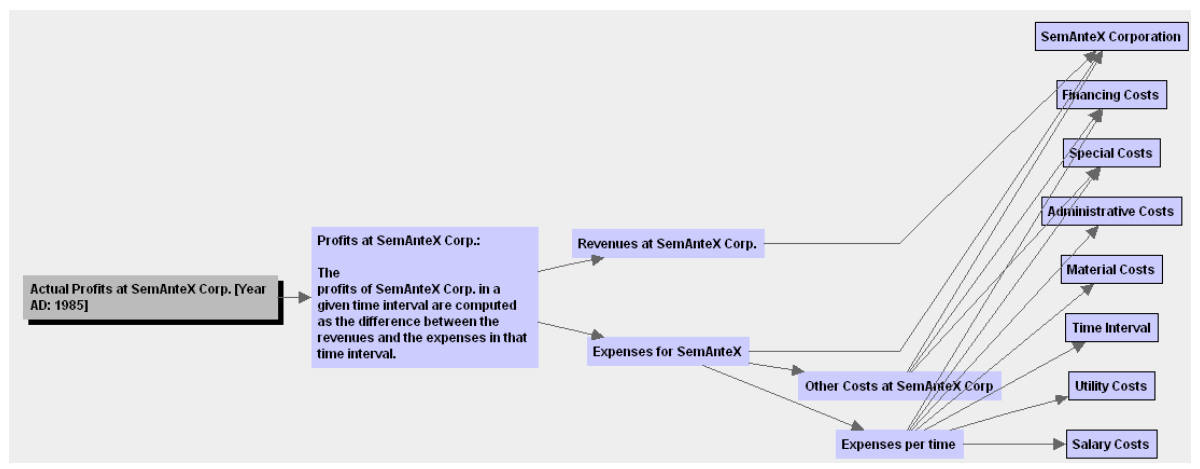

Fig. 3. Navigating the Spreadsheet Intention

In Figure 2 we have already shown the main control panel of the SACHS system in the right bottom hand corner. This allows the user to enable various semantic enhancements in the spreadsheet and also search the intention semantically. From a semantic point of view, contextual views of the spreadsheet intention as the one in Figure 3 are probably most interesting and helpful. Such views allow a user to understand the connections between spreadsheet cells and background information. This view aggregates information about 
- the intention of a cell in terms of the intended function of its functional block, and the intended arguments.

- how the cell value is computed from the values of its arguments.

- and the intentions of the arguments.

All information points in the pop-up are hyperlinked to their respective sources in the OMDoc document or in the spreadsheet so that the graph view can also serve as a meaning-driven navigation tool. In a future version of SACHS, these hyperlinks could pre-instantiate the intention model with the argument values and allow an exploration from the view of the current cell - in our example in Figure 3 the intention specialized to the year 1985. Note that our example here only shows the situation for a formula-computed cell. For other provenances, the pop-up would visualize the provenance object given by the formula interpretation. For instance, for cell [B9] the provenance object is "imported from Salaries.xls[B5]", so we can visualize the data from that cell using the existing SACHS pop-up.

As OMDoc is organized by theories, the provision of multi-level theory graphs as in the CPOINT system [Koh05a] are nice-to-have services one can think of.

\subsection{Evaluation: Estimating the Semantic Overhead in SACHS}

As always the interesting question at the end of the implementation of exciting new ideas is whether it was worth the effort. Even though we can't answer that yet objectively, we can at least give an estimate for the costs of the semantic overhead and its reuse.

At the moment, our approach presupposes that the spreadsheet author documents the spreadsheet intention as an OMDoc document and also annotates the spreadsheet with interpretation information. Both tasks place a heavy burden on the author and currently restrict our approach to mission-critical or very complex spreadsheets.

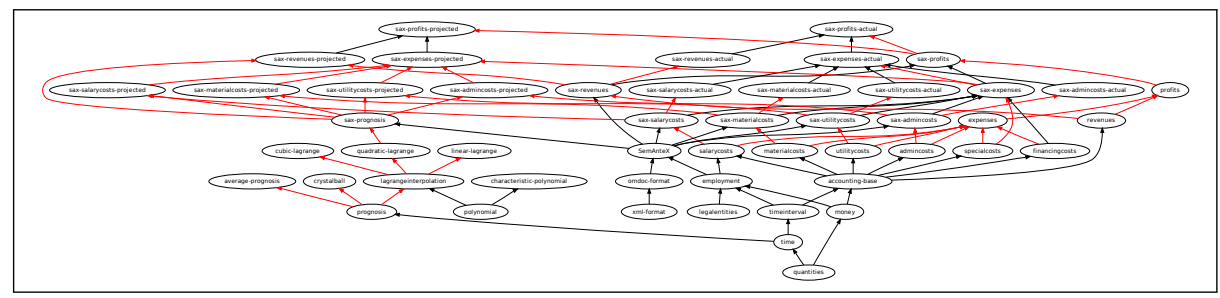

Fig. 4. The Theory Graph of Background Knowledge for our Example Spreadsheet

For our example we have semi-formalized the background knowledge in a collection of 47 theories (see Figure 4), which serve as the source of the new SACHS services reported here. Of these, 27 theories contain general knowledge about quantities, units, basic cost accounting, and prognosis. Only 20 were developed specially for our example: a theory about the SemAnteX Corp. itself and 
union theories specializing accounting and prognosis to the SemAnteX Corp. peculiarities. A dozen general theories about real arithmetic were not taken into account here, since they were available externally.

By size, the specific theories only amounted to ca. $25 \%$ of the semi-formalization. We are currently developing a background knowledge corpus for a central part of the DFKI controlling system, which has three orders of magnitude more cells than the expository example in this paper. But the semi-formalization of this is only double in size, conceivably since the controlling system is relatively regular, for instance it is organized in about 20 analogous sheets for the respective DFKI departments.

In general, we expect the burden of specifying the ontology to decrease as more and more OMDoc content dictionaries for common models (e.g. standard accounting techniques) appear. Our case study has shown that for an efficient development of background ontologies we need to integrate editing facilities into the graph view in Figure 3. For the interpretations, we plan to adapt techniques of header, unit, and template inference [AE04,AE06] to partially automate the annotation process via suggestions.

For evaluating the usefulness of the SACHS system we are currently undertaking a formal user study on the DFKI controlling system - our expository example in Figure 2 is too simple to require a help system really. First informal feedback was encouraging, for instance, users highly appreciated being made aware of the differing reference periods data that were implicit in the concrete spreadsheet layout.

\section{Conclusion and Outlook}

We have analyzed the reasons for users' difficulties in understanding and appropriating complex spreadsheets, as they are found e.g. in financial controlling systems. We claim that the ultimate cause is that spreadsheets are weak as active documents, because their underlying semantic documents are biased to computational aspects and fail to model the provenance, interpretation, and ontological relations of the objects and concepts operationalized by the system. To remedy the situation we propose to explicitly model the intention of a spreadsheet as an intention model in a collection of OMDoc content dictionaries which serve as an explicit knowledge base for the spreadsheet. Finally, we present the workin-progress SACHS system that draws on such intention models to offer various semantic services that aid the user in understanding and interacting with the spreadsheets.

In essence, our approach makes double use of the following duality identified by Fensel in [Fen08]

- Ontologies define formal semantics for information, consequently allowing information processing by a computer.

- Ontologies define real-world semantics, which makes it possible to link machine processable content with meaning for humans based on consensual terminologies. 
In the analysis we look at the formal semantics of spreadsheets and find a computational bias that hampers understanding since it fails to model consensual terminologies and therefore leads to real-world usability problems. In our proposed solution we extend the formal semantics of spreadsheets to draw on explicitly represented consensual terminologies.

While a semantic help system for a spreadsheet-based controlling system was the original motivation for our analysis, we feel that an explicit representation of the intention model of a spreadsheet has many more applications: it can be used for verification of the formulae, for change management along the lines of [MW07], and automated generation of user-adapted spreadsheets.

Our approach seems to be related to "Class Sheets" [EE05] introduced by ENGELS and ERWIG. Their class descriptions can also be counted as an external, structured intention model, which is referenced by an interpretation mapping. We will have to study the relation to our work more closely, but it seems that their work suffers the same computational bias as the spreadsheets themselves. But classes with extensive documentation or UML diagrams might go some ways towards generating a help system like SACHS.

In the future we plan to extend the repertoire of semantic services of the SACHS system. For instance, we envision a dual service to the one in Figure 3 which could visualize where the value of a cell is used to get an intuition for the relevance of a cell value.

As a theoretical extension, we can see the interpretation mappings as logic morphisms [RK09], and logic morphisms in the domain knowledge as mathematical framings (ways to view mathematical objects in terms of well-understood ones). This allow us to re-use much of the higher-level MKM techniques and OMDoc functionalities based on theory graphs: we have already extended the user interaction of the SACHS to be framing-aware [KK09], which allows the user to customize the interaction to her subjective background and goals.

But future extensions are not limited to spreadsheets. Note that the semantic analysis in Section 2 is largely independent of the computational information that is at the heart of spreadsheets. The semantic information we are after only pertains to the use of data grids as a user interface to complex functions. In the future we plan to generalize the intention model architecture presented in Section 3 to the case of data grids - e.g. tables of experimental results or raster data from satellite images. Moreover, we want to develop a native infrastructure for representing "data grids" as a user interaction feature in OMDoc: In accordance with the format, the provenance and interpretation functionality would allow to link grid presentations to their intention without leaving the language. OMDoc-based systems could then pick up the semantics and offer complex interactions based on them. This would lead to much more general active documents. We could even turn the approach presented in this paper around and generate SACHS spreadsheets from OMDoc documents as active documents.

Acknowledgements This paper contains our view on the foundations of the SACHS project which aims to extend semantic document modeling to spreadsheets. Bernd Krieg-Brückner has been proposing for years to do this. He always 
maintained that spreadsheets would be ideal targets for semantic preloading. Indeed our research shows that spreadsheets are semantically much more interesting than anticipated. We also thank the other SACHS project members: Dieter Hutter, Achim Mahnke, as well as Klaus Hofmann for valuable discussions.

\section{References}

$\left[\mathrm{ABC}^{+}\right.$03] Ron Ausbrooks, Stephen Buswell, David Carlisle, Stéphane Dalmas, Stan Devitt, Angel Diaz, Max Froumentin, Roger Hunter, Patrick Ion, Michael Kohlhase, Robert Miner, Nico Poppelier, Bruce Smith, Neil Soiffer, Robert Sutor, and Stephen Watt. Mathematical Markup Language (MathML) version 2.0 (second edition). W3C recommendation, World Wide Web Consortium, 2003.

[AE04] Robin Abraham and Martin Erwig. Header and unit inference for spreadsheets through spatial analysis. In IEEE International Symposium on Visual Languages and Human-Centric Computing, pages 165-172, 2004.

[AE06] Robin Abraham and Martin Erwig. Inferring templates from spreadsheets. In ICSE '06: Proceedings of the 28th international conference on Software engineering, pages 182-191, New York, NY, USA, 2006. ACM.

$\left[\mathrm{BCC}^{+} 04\right]$ Stephen Buswell, Olga Caprotti, David P. Carlisle, Michael C. Dewar, Marc Gaetano, and Michael Kohlhase. The Open Math standard, version 2.0. Technical report, The Open Math Society, 2004.

[BSRR02] M. M. Burnett, A. Sheretov, B. Ren, and G. Rothermel. Testing homogenous spreadsheet grids with the "what you see is what you test" methodology. IEEE Transactions on Software Engineering, 29(6):576-594, 2002.

[CoF04] CoFI (The Common Framework Initiative). CASL Reference Manual. LNCS 2960 (IFIP Series). Springer, 2004.

[EE05] Gregor Engels and Martin Erwig. ClassSheets: Automatic generation of spreadsheet applications from object oriented specifications. In $20^{\text {th }}$ IEEE/ACM International Conference on Automated Seofware Engineering, pages 124-155. IEEE Computer Society, 2005.

[Fen08] Dieter Fensel. Foreword. In Martin Hepp, Pieter De Leenheer, Aldo de Moor, and York Sure, editors, Ontology Management: Semantic Web, Semantic Web Services, and Business Applications, Semantic Web and beyond: Computing for Human Experience, pages 9-11. Springer Verlag, 2008.

[GS99] Brian R. Gaines and Mildred L. G. Shaw. Enbedding formal knowledge models in active documents; creating problem-solving documents. Communications of the ACM, 42(1):57-63, 1999.

[HKS06] Eberhard Hilf, Michael Kohlhase, and Heinrich Stamerjohanns. Capturing the content of physics: Systems, observables, and experiments. In Jon Borwein and William M. Farmer, editors, Mathematical Knowledge Management, MKM'06, number 4108 in LNAI, pages 165-178. Springer Verlag, 2006.

[HM00] E. Heinrich and H. Maurer. Active documents: Concept, implementation, and applications. Journal of Universal Computer Science, 6(12):1197-1202, 2000 .

[KK09] Andrea Kohlhase and Michael Kohlhase. Spreadsheet interaction with frames: Exploring a mathematical practice. This Volume.

[Koh05a] Andrea Kohlhase. Cpoint, 2005. http://kwarc.info/projects/CPoint/. 
[Koh05b] Andrea Kohlhase. Overcoming Proprietary Hurdles: CPoint as Invasive Editor. In Fred de Vries, Graham Attwell, Raymond Elferink, and Alexandra Tödt, editors, Open Source for Education in Europe: Research and Practise, pages 51-56, Heerlen, The Netherlands, November 2005. Open Universiteit Nederland, Open Universiteit Nederland. Proceedings at http://hdl. handle.net/1820/483.

[Koh06] Michael Kohlhase. OMDoc - An open markup format for mathematical documents [Version 1.2]. Number 4180 in LNAI. Springer Verlag, 2006.

$\left[\mathrm{MGM}^{+}\right.$08] Luc Moreau, Paul Groth, Simon Miles, Javier Vazquez, John Ibbotson, Sheng Jiang, Steve Munroe, Omer Rana, Andreas Schreiber, Victor Tan, and Laszlo Varga. The provenance of electronic data. Communications of the ACM, 51(4):52-58, 2008.

[MvH04] Deborah L. McGuinness and Frank van Harmelen. OWL web ontology language overview. W3C recommendation, W3C, February 2004.

[MW07] Normen Müller and Marc Wagner. Towards Improving Interactive Mathematical Authoring by Ontology-driven Management of Change. In Alexander Hinneburg, editor, Wissens- und Erfahrungsmanagement LWA (Lernen, Wissensentdeckung und Adaptivität) conference proceedings, pages 289-295. Martin-Luther-University Halle-Wittenberg, 2007.

[Pan00] Raymond R. Panko. Spreadsheet errors: What we know. what we think we can do. In Symp. of the European Spreadsheet Risks Interest Group (EuSpRIG), 2000.

[RK09] Florian Rabe and Michael Kohlhase. A web-scalable module system for mathematical theories. Manuscript, to be submitted to the Journal of Symbolic Computation, 2009.

$\left[\mathrm{UCI}^{+} 06\right]$ Victoria Uren, Philipp Cimiano, José Iria, Siegfried Handschuh, Maria Vargas-Vera, Enrico Motta, and Fabio Ciravegna. Semantic annotation for knowledge management: Requirements and a state of the art. Web Semantics: Science, Services, and Agents on the World Wide Web, 4(1):14-28, Jan. 2006.

[W3C08] RDFa Primer. http://www.w3.org/TR/xhtml-rdfa-primer/, 2008.

[Win06] Terry Winograd. The spreadsheet. In Terry Winograd, John Bennett, Laura de Young, and Bradley Hartfield, editors, Bringing Design to Software, pages 228-231. Addison-Wesley, 1996 (2006).

[Wol02] Stephen Wolfram. The Mathematica Book. Cambridge University Press, 2002 . 